\title{
Estimation of free-living energy expenditure using a novel activity monitor designed to minimize obtrusiveness.
}

Citation for published version (APA):

Bonomi, A., Plasqui, G., Goris, A. H., \& Westerterp, K. R. (2010). Estimation of free-living energy expenditure using a novel activity monitor designed to minimize obtrusiveness. Obesity, 18(9), $1845-1851$. https://doi.org/10.1038/oby.2010.34

Document status and date:

Published: 01/09/2010

DOI:

10.1038/oby.2010.34

Document Version:

Publisher's PDF, also known as Version of record

Document license:

Taverne

Please check the document version of this publication:

- A submitted manuscript is the version of the article upon submission and before peer-review. There can be important differences between the submitted version and the official published version of record.

People interested in the research are advised to contact the author for the final version of the publication, or visit the DOI to the publisher's website.

- The final author version and the galley proof are versions of the publication after peer review.

- The final published version features the final layout of the paper including the volume, issue and page numbers.

Link to publication

\footnotetext{
General rights rights.

- You may freely distribute the URL identifying the publication in the public portal. please follow below link for the End User Agreement:

www.umlib.nl/taverne-license

Take down policy

If you believe that this document breaches copyright please contact us at:

repository@maastrichtuniversity.nl

providing details and we will investigate your claim.
}

Copyright and moral rights for the publications made accessible in the public portal are retained by the authors and/or other copyright owners and it is a condition of accessing publications that users recognise and abide by the legal requirements associated with these

- Users may download and print one copy of any publication from the public portal for the purpose of private study or research.

- You may not further distribute the material or use it for any profit-making activity or commercial gain

If the publication is distributed under the terms of Article $25 \mathrm{fa}$ of the Dutch Copyright Act, indicated by the "Taverne" license above, 


\title{
Estimation of Free-Living Energy Expenditure Using a Novel Activity Monitor Designed to Minimize Obtrusiveness
}

\author{
Alberto G. Bonomi ${ }^{1,2}$, Guy Plasqui ${ }^{1}$, Annelies H.C. Goris ${ }^{3}$ and Klass R. Westerterp ${ }^{1}$
}

The aim of this study was to investigate the ability of a novel activity monitor designed to be minimally obtrusive in predicting free-living energy expenditure. Subjects were 18 men and 12 women (age: $41 \pm 11$ years, BMl:

$24.4 \pm 3 \mathrm{~kg} / \mathrm{m}^{2}$ ). The habitual physical activity was monitored for 14 days using a DirectLife triaxial accelerometer for movement registration $\left(\right.$ Tracmor $_{\mathrm{D}}$ ) (Philips New Wellness Solutions, Lifestyle Incubator, the Netherlands). Tracmor $_{\mathrm{D}}$ output was expressed as activity counts per day (Cnts/d). Simultaneously, total energy expenditure (TEE) was measured in free-living conditions using doubly labeled water (DLW). Activity energy expenditure (AEE) and the physical activity level (PAL) were determined from TEE and sleeping metabolic rate (SMR). A multiple-linear regression model predicted $76 \%$ of the variance in TEE, using as independent variables SMR (partial- $r^{2}=0.55, P<0.001$ ), and Cnts $/ \mathrm{d}$ (partial- $r^{2}=0.21, P<0.001$ ). The s.e. of TEE estimates was $0.9 \mathrm{MJ} /$ day or $7.4 \%$ of the average TEE. A model based on body mass (partial- $r^{2}=0.31, P<0.001$ ) and Cnts/d (partial- $r^{2}=0.23, P<0.001$ ) predicted $54 \%$ of the variance in TEE. Cnts/d were significantly and positively associated with AEE $(r=0.54, P<0.01)$, PAL $(r=0.68$, $P<0.001)$, and AEE corrected by body mass $(r=0.71, P<0.001)$. This study showed that the Tracmor is a highly accurate instrument for predicting free-living energy expenditure. The miniaturized design did not harm the ability of the instrument in measuring physical activity and in determining outcome parameters of physical activity such as TEE, AEE, and PAL.

Obesity (2010) 18, 1845-1851. doi:10.1038/oby.2010.34

\section{INTRODUCTION}

The increasing incidence of obesity represents one of the greatest health challenges of the current century (1). Obesity is a serious threat to health because it increases the risk of developing many chronic diseases, such as diabetes and cardiovascular disease (2). Several studies have highlighted the relationship between obesity and genetic factors (3), biological, socioeconomic, and environmental factors (4). However, the main underlying cause of obesity is represented by a chronic imbalance between energy intake and energy expenditure. Achieving a negative balance between energy intake and expenditure is a key factor to lose weight. This could be realized, for example, by increasing energy expenditure through physical activity. Furthermore, increasing the level of physical activity has been often recommended to improve health and reduce risks for chronic diseases (5). For these reasons, accurate measurements of physical activity and energy expenditure are necessary to define optimal intervention strategies to achieve health benefits.

Several methods have been proposed to monitor physical activity and to determine total energy expenditure
(TEE). The gold standard technique for the assessment of TEE in free-living conditions is the doubly labeled water method (DLW), which is based on indirect calorimetry. However, DLW is markedly expensive and requires appropriate laboratory equipment for samples analysis. Thus, it is infrequently used in large-population studies to assess TEE. Other methods such as diaries and questionnaires (6), heart rate monitors (7), and motion sensors (8) have been proposed to assess physical activity and to estimate TEE. Ideally, physical activity should be accurately measured in free-living conditions, over a period of time representative of the habitual activity level, and with minimal discomfort to the subject. Activity monitors based on accelerometers reasonably satisfy these requirements, and therefore have been used in several studies. Accelerometer output has been often used to estimate, together with subjects' characteristics, the TEE, and the activity energy expenditure (AEE) $(9,10)$. Furthermore, accelerometers' output is linearly related to the physical activity level (PAL, TEE/sleeping, or resting metabolic rate) (10). 
Improving the accuracy of accelerometers in predicting TEE, AEE, and PAL has been the focus of several studies (11-14). Frequently, the achievement of higher estimation accuracy coincided with the increase in the complexity of the measurement system. For example, network systems of accelerometers, placed in different body parts, have been developed to improve the accuracy in measuring TEE and AEE $(11,13,15)$. Likewise, accelerometers have been coupled to heart rate monitors to increase the accuracy in the AEE estimation $(7,16)$. However, the performance of these complex systems showed a relatively limited improvement in the estimation error of TEE (10). Furthermore, they have seldom been validated in free-living conditions using, as a reference, DLW. On the other hand, activity monitors used in daily life should be minimally obtrusive. This implies the employment of a simple measuring system, with small dimensions, and characterized by long battery life and memory storage capacity. This would be beneficial to improve the compliance of the users to the measuring device, and to reduce the interference of the monitoring system with the spontaneous activity behavior. However, unobtrusive accelerometers designed to quantify physical activity using activity counts, often showed poor correlation with AEE and PAL (10).

The present study investigated the ability of a novel accelerometer specifically designed to be minimally obtrusive, the DirectLife triaxial accelerometer for movement registration (Philips New Wellness Solutions), to predict TEE, AEE, and PAL.

\section{METHODS AND PROCEDURES}

\section{Subjects}

The study population was composed of 30 healthy adults (18 men and 12 women). They were recruited by advertisement in local newspapers to participate in the study. Written informed consent was obtained and the study was approved by the Ethics Committee of the Maastricht University Medical Centre.

\section{Study design}

Subjects reported to the laboratory on day 0 at 9:00 PM for an overnight stay in a respiration chamber. Anthropometric measurements were taken in the morning after an overnight fast. Body mass was measured on an electronic scale (Mettler Toledo ID1 Plus; Mettler Toledo, Giessen, Germany) to the nearest $0.01 \mathrm{~kg}$. Height was measured to the nearest $0.1 \mathrm{~cm}$ (SECA Model 220; SECA, Hamburg, Germany). Body volume was determined by underwater weighing. During the underwater weighing the residual lung volume was measured using the helium dilution technique (Volugraph 2000; Mijnhardt, Bunnik, the Netherlands). Total body water was determined using deuterium dilution, according to the Maastricht protocol (17). Body composition was calculated from body mass, body volume, and total body water using the Siri's three-compartment model (18). The study included a 2 -week observation period for the measurements of energy expenditure, from the morning of day 1 until the morning of day 15. Simultaneously, physical activity was monitored using a triaxial accelerometer for movement registration.

\section{Sleeping metabolic rate (SMR)}

SMR was measured during an overnight stay in the respiration chamber. The room consisted in an airtight chamber with an internal volume of $14 \mathrm{~m}^{3}$ and was furnished with bed, table, chair, freeze toilet, washing bowl, radio, television, and a computer (19). Energy expenditure was calculated from $\mathrm{O}_{2}$-consumption and $\mathrm{CO}_{2}$-production according to Weir's formula (20). SMR was defined as the lowest observed energy expenditure for three consecutive hours during the night. Room temperature was held constant at $20 \pm 1^{\circ} \mathrm{C}$.

\section{Energy expenditure}

The TEE was measured using DLW according to the Maastricht protocol (17). On the evening of day 0 , after the collection of a background urine sample, subjects drank a weighted amount of ${ }^{2} \mathrm{H}_{2}{ }^{18} \mathrm{O}$ such that baseline levels were increased with 100 p.p.m. for ${ }^{2} \mathrm{H}$ and 200 p.p.m. for ${ }^{18} \mathrm{O}$. Additionally, urine samples were collected in the morning (from second voiding) of day 1 , day 8 , and day 15 , and in the evening of day 1 , day 7 , and day 14 . The AEE was measured as $(0.9 \times$ TEE $)-$ SMR, assuming the diet-induced thermogenesis to be $10 \%$ of TEE (21). The mean PAL was calculated as TEE/SMR. AEE was also expressed per $\mathrm{kg}$ of body mass as commonly proposed to describe physical activity (22-24).

\section{Physical activity monitoring}

The habitual physical activity was assessed using a DirectLife triaxial accelerometer for movement registration $\left(\right.$ Tracmor $_{D}$ ) (Philips New Wellness Solutions; http://www.directlife.philips.com), which was based on the research device Tracmor $(10,24)$. The device is a small $(3.2 \times 3.2 \times$ $0.5 \mathrm{~cm})$, light-weight $(12.5 \mathrm{~g})$ instrument (Figure 1). The Tracmor $_{\mathrm{D}}$ is waterproof up to $30 \mathrm{~m}$ depth, and has a battery life of 3 weeks and an internal memory that can store data for up to 22 weeks. The features of the Tracmor ${ }_{D}$ have been designed to enhance wearability and reduce the interference of the monitoring system with the spontaneous activity behavior. This has been shown in a study by Goris and Holmes (25), where the Tracmor ${ }_{D}$ was used to monitor physical activity in a population of 217 subjects. After 12 weeks from the start of the monitoring period still 140 subjects were wearing the device on a daily basis. The Tracmor $_{D}$ is also able to provide feedback to the user on the performed physical activity. An indicator bar of light-emitting diodes activates on demand, showing the achievement of the day in terms of amount of physical activity performed as compared to an optimal target defined based on recent recommendations of the World Health Organization. This function was temporarily disabled by the manufacturer in the Tracmor $_{D}$ employed in the present study. The Tracmor ${ }_{D}$ was placed at the lower back using an elastic belt, and the subjects were instructed to wear the accelerometer during waking hours. A diary was used to report periods in which the subject was not wearing the Tracmor $_{D}$ during the day. At the end of the monitoring period the Tracmor ${ }_{D}$ was connected to a personal computer and the recorded data were downloaded using dedicated software (Philips New Wellness Solutions). Tracmor ${ }_{D}$ output

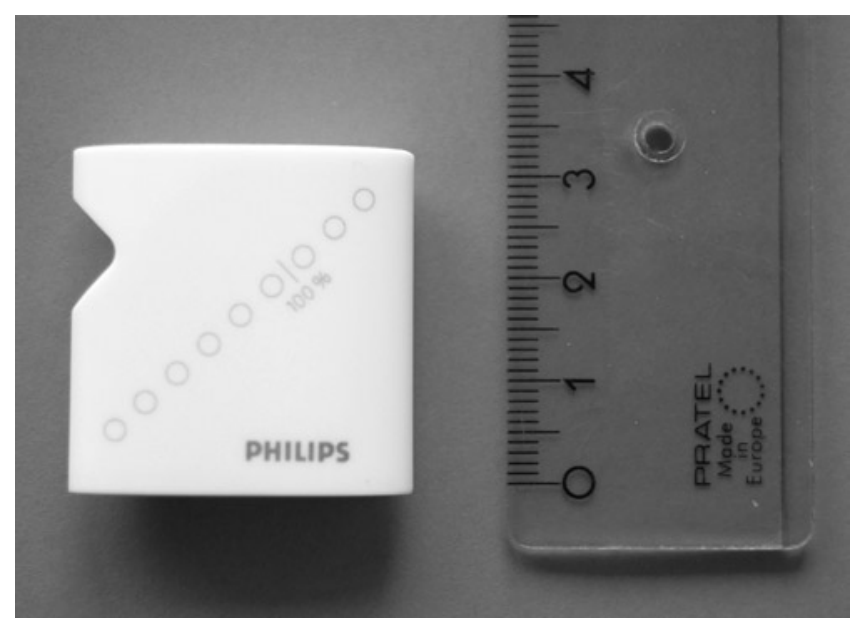

Figure 1 The DirectLife activity monitor $\left(\right.$ Tracmor $\left._{D}\right)$. The figure shows the small dimensions of the instrument (left) as compared to a centimeter ruler (right). 
was expressed as activity counts/minute. The Tracmor ${ }_{D}$ activity counts/ minute were summed over the entire monitoring period and divided by the number of monitoring days to determine the average Tracmor ${ }_{D}$ counts per day (Cnts/d).

\section{Statistical analysis}

Stepwise multiple-linear regression analysis was used to select the best independent variables to predict TEE and AEE. The Cnts/d was the independent variable that was considered to account for differences in physical activity. Additionally, three different sets of independent variables were considered to account for the differences in body size: SMR, basic body characteristics (body mass, height, age, and gender) and advanced body characteristics (fat mass, fat-free mass, age, gender). The independent variables considered in the regression analysis of AEE were the same as in the regression analysis of TEE with the exception of SMR. Simple linear regression was used to develop prediction models for PAL, and AEE scaled to body mass (AEE/kg) using as independent variable $\mathrm{Cnts} / \mathrm{d}$. The leave-one-subject-out crossvalidation was used to determine the standard error of validation (SEV), representing the

Table 1 Subjects' characteristics $(N=30)$

\begin{tabular}{|c|c|c|}
\hline Parameter & Mean \pm s.d. & Range \\
\hline$N(\mathrm{M} / \mathrm{F})$ & $30(18 / 12)$ & \\
\hline Age, years & $41 \pm 11$ & $26-60$ \\
\hline Body mass, kg & $74.4 \pm 11.9$ & 51.9-103.4 \\
\hline Height, m & $1.74 \pm 0.08$ & $1.58-1.89$ \\
\hline $\mathrm{BMI}, \mathrm{kg} / \mathrm{m}^{2}$ & $24.4 \pm 3.0$ & $19.0-31.4$ \\
\hline $\mathrm{FM}, \mathrm{kg}$ & $19.4 \pm 6.5$ & $8.4-33.2$ \\
\hline FFM, kg & $54.9 \pm 8.4$ & $39.4-70.2$ \\
\hline SMR, MJ/day & $6.9 \pm 0.8$ & $5.5-8.3$ \\
\hline TEE, MJ/day & $12.1 \pm 1.8$ & $8.7-15.5$ \\
\hline AEE, MJ/day & $4.0 \pm 1.2$ & $2.1-6.4$ \\
\hline PAL & $1.76 \pm 0.17$ & $1.43-2.08$ \\
\hline Tracmor $_{D}, 10^{3}$ counts/day & $1,572 \pm 450$ & $877-2,626$ \\
\hline
\end{tabular}

AEE, activity energy expenditure; FFM, fat-free mass; FM, fat mass; PAL, physical activity level; SMR, sleeping metabolic rate; TEE, total energy expenditure; Tracmor $_{D}$, DirectLife activity monitor output. performance of the models in estimating TEE, AEE, PAL, and AEE/ body mass for subjects not used to develop the prediction equations. The Pearson correlation coefficient $(r)$ was used to describe the association between variables. The measured parameters are expressed as mean \pm s.d. The statistical software SigmaStat (Systat Software, San Jose, CA) was used for statistical analysis. The significance level was set to $P<0.05$.

\section{RESULTS}

Physical characteristics of the subjects are presented in Table 1. Subjects wore the accelerometer on average $14.4 \pm 1.2 \mathrm{~h} / \mathrm{day}$, which was $95 \pm 3 \%$ of their waking hours. Measured TEE, AEE, AEE/kg, and PAL are presented in Table 1. The Cnts/d were significantly associated with $\operatorname{AEE}(r=0.54, P<0.01)$, $\mathrm{AEE} / \mathrm{kg}(r=0.71, P<0.001)$, and PAL $(r=0.68, P<0.001)$. No significant association was observed between TEE and Cnts/d $(P=0.16)$.

\section{Prediction of TEE}

The Cnts/d significantly contributed to the explained variance in TEE of each prediction model developed using the multiple-linear regression analysis (Table 2). The model based on SMR and Cnts/d explained 76\% $(r=0.87)$ of the variation in TEE, with a standard error of estimate (SEE) of $0.9 \mathrm{MJ} /$ day or $7.4 \%$ of the mean measured TEE. The residuals of the model were negatively associated with the measured TEE $(r=-0.49, P<0.05)$ (Figure 2). Considering basic body characteristics and Cnts/d in the stepwise regression analysis, only body mass and Cnts/d were included in the prediction model, and the explained variation in TEE was $54 \%(r=0.73)$. The residuals of this model were significantly and negatively associated with the measured TEE $(r=-0.68$, $P<0.001)$. When advanced body characteristics and Cnts/d were used in the stepwise regression analysis, fat-free mass and Cnts/d were included in the prediction model of TEE. The explained variation in TEE of this model was 68\% $(r=$ 0.82 ). The residuals of this model were negatively associated with the measured TEE $(r=-0.57, P<0.05)$. None of the

Table 2 Prediction models of TEE

\begin{tabular}{|c|c|c|c|c|c|c|c|c|c|}
\hline Dependent & Independent & Coefficient & $P$ & Par $r^{2}$ & s.d. & $r^{2}$ & SEE & SEV & SEV\% \\
\hline \multirow[t]{2}{*}{ TEE } & Intercept & -4.00 & & & 0.35 & & & & \\
\hline & SMR & 1.9 & $<0.001$ & 0.55 & 0.05 & & & & \\
\hline Model & & & & & & $76 \%$ & $0.90 \mathrm{MJ} /$ day & $0.96 \mathrm{MJ} /$ day & $7.9 \%$ \\
\hline \multirow[t]{2}{*}{ TEE } & Intercept & 0.65 & & & 0.4 & & & & \\
\hline & Cnts/d & $2 \cdot 10^{-6}$ & $<0.001$ & 0.23 & $0.1 \cdot 10^{-6}$ & & & & \\
\hline Model & & & & & & $54 \%$ & $1.27 \mathrm{MJ} /$ day & $1.35 \mathrm{MJ} /$ day & $10.5 \%$ \\
\hline \multirow[t]{3}{*}{ TEE } & Intercept & 0.04 & & & 0.3 & & & & \\
\hline & FFM & 0.17 & $<0.001$ & 0.51 & 0.005 & & & & \\
\hline & Cnts/d & $1.67 \cdot 10^{-6}$ & $<0.001$ & 0.17 & $0.08 \cdot 10^{-6}$ & & & & \\
\hline
\end{tabular}

Cnts/d, triaxial accelerometer for movement registration output; FFM, fat-free mass; $P$, significance level; Par $r^{2}$, partial correlation; $r^{2}$, correlation coefficient of the model; s.d., standard deviation of the coefficient of the model at each step of the crossvalidation; SEE, standard error of estimation of the model; SEV\%, percentage SEV of the average dependent variable; SEV, standard error of validation of the model; SMR, sleeping metabolic rate; TEE, total daily energy expenditure. 


\section{METHODS AND TECHNIQUES}

physical characteristics of the population were correlated to the residual of the prediction models. Coefficients, significance level, partial correlations, SEE, and SEV of all models are summarized in Table 2.

\section{Prediction models of AEE}

The Cnts/d significantly contributed to the explained variance in AEE of each developed prediction model (Table 3). Considering advanced body characteristics and $\mathrm{Cnts} / \mathrm{d}$ in the stepwise regression analysis, fat-free mass, and Cnts/d were included in the prediction model. The explained variation in AEE was $53 \%(r=0.73)$ with a SEE of $0.82 \mathrm{MJ} /$ day or $20.5 \%$. The residuals of this model were negatively associated with the
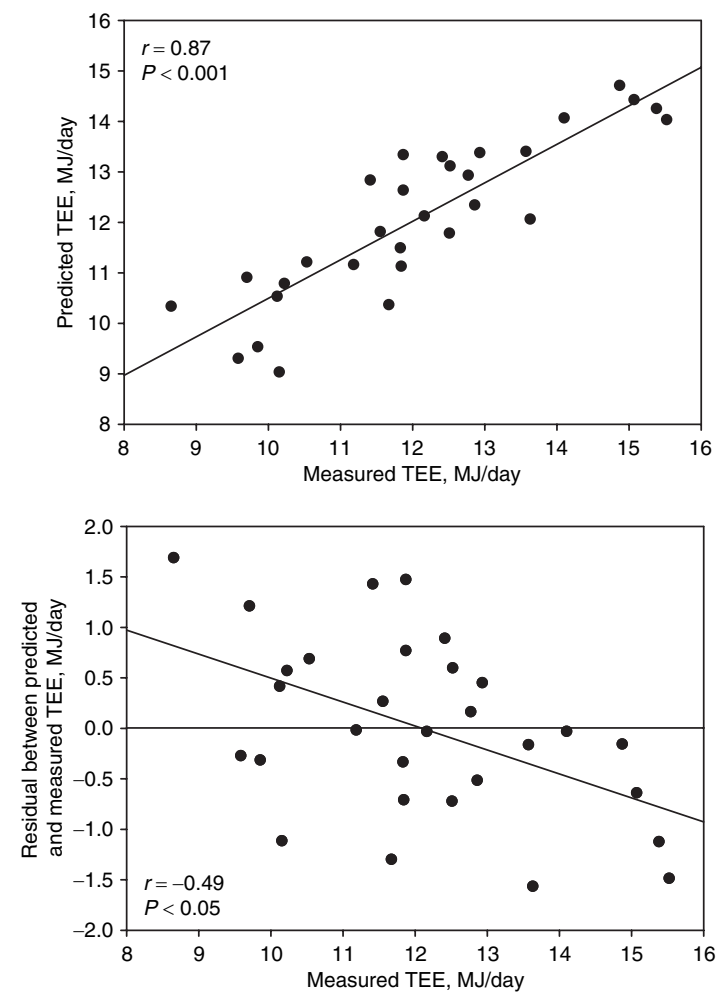

Figure 2 Regression and residual plots of the prediction models of total energy expenditure (TEE) based on accelerometer output and SMR. SMR, sleeping metabolic rate. measured AEE $(r=-0.69, P<0.05)$ (Figure 3$)$. When basic body characteristics and Cnts/d were entered as independent variables in the stepwise regression analysis, body mass and Cnts/d, significantly contributed to the explained variation in AEE. The model explained $46 \%(r=0.68)$ of the variation in AEE. The residuals of this model were negatively associated with the measured AEE $(r=-0.73, P<0.001)$. Coefficients, significance level, partial correlations, SEE, and SEV of all models are summarized in Table 3.

\section{Prediction models of PAL and AEE/kg}

Simple linear regression was used to develop prediction models of PAL and AEE/kg using Cnts/d, as these represent common
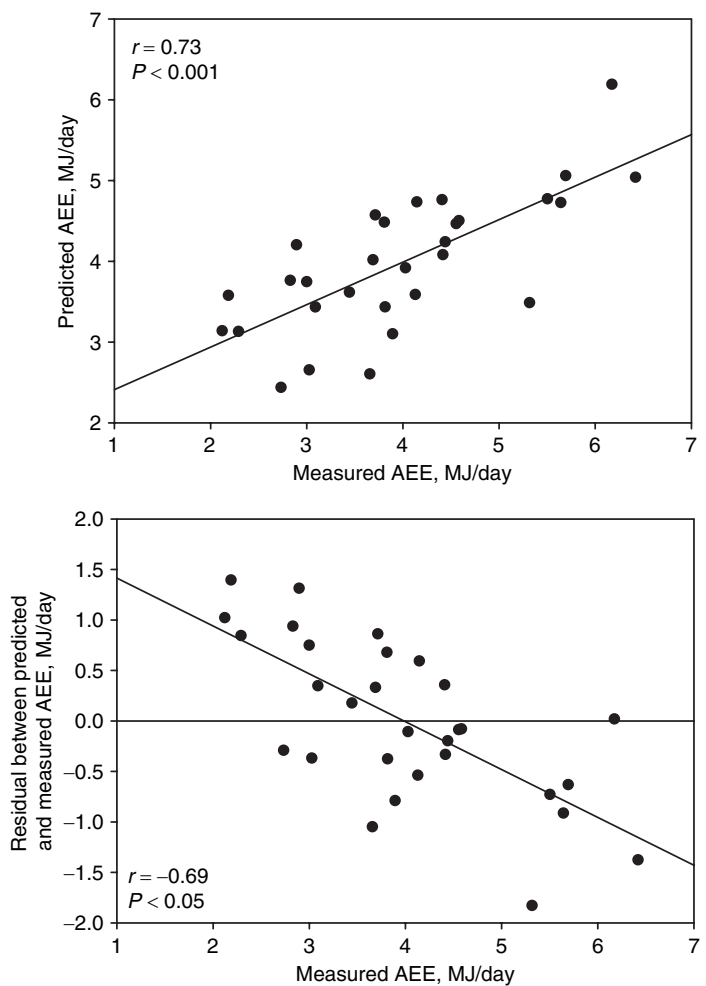

Figure 3 Regression and residual plots of the prediction models of activity energy expenditure (AEE) based on accelerometer output and fat-free mass.

Table 3 Prediction models of AEE

\begin{tabular}{|c|c|c|c|c|c|c|c|c|c|}
\hline Dependent & Independent & Coefficient & $P$ & Par $r^{2}$ & s.d. & $r^{2}$ & SEE & SEV & SEV\% \\
\hline \multirow[t]{2}{*}{ AEE } & Intercept & -1.89 & & & 0.31 & & & & \\
\hline & Body mass & 0.04 & $<0.01$ & 0.30 & 0.003 & & & & \\
\hline Model & & & & & & $46 \%$ & $0.94 \mathrm{MJ} /$ day & $0.88 \mathrm{MJ} /$ day & $22 \%$ \\
\hline \multirow[t]{2}{*}{ AEE } & Intercept & -2.30 & & & 0.25 & & & & \\
\hline & Cnts/d & $1.6 \cdot 10^{-6}$ & $<0.001$ & 0.23 & $0.06 \cdot 10^{-6}$ & & & & \\
\hline Model & & & & & & $53 \%$ & $0.87 \mathrm{MJ} /$ day & $0.82 \mathrm{MJ} /$ day & $20 \%$ \\
\hline
\end{tabular}

AEE, activity energy expenditure; Cnts/d, triaxial accelerometer for movement registration output; FFM, fat-free mass; $P$, significance level; Par $r^{2}$, partial correlation; $r^{2}$, correlation coefficient of the model; s.d., standard deviation of the coefficient of the model at each step of the crossvalidation; SEE, standard error of estimation of the model; SEV\%, percentage SEV of the average dependent variable; SEV, standard error of validation of the model. 

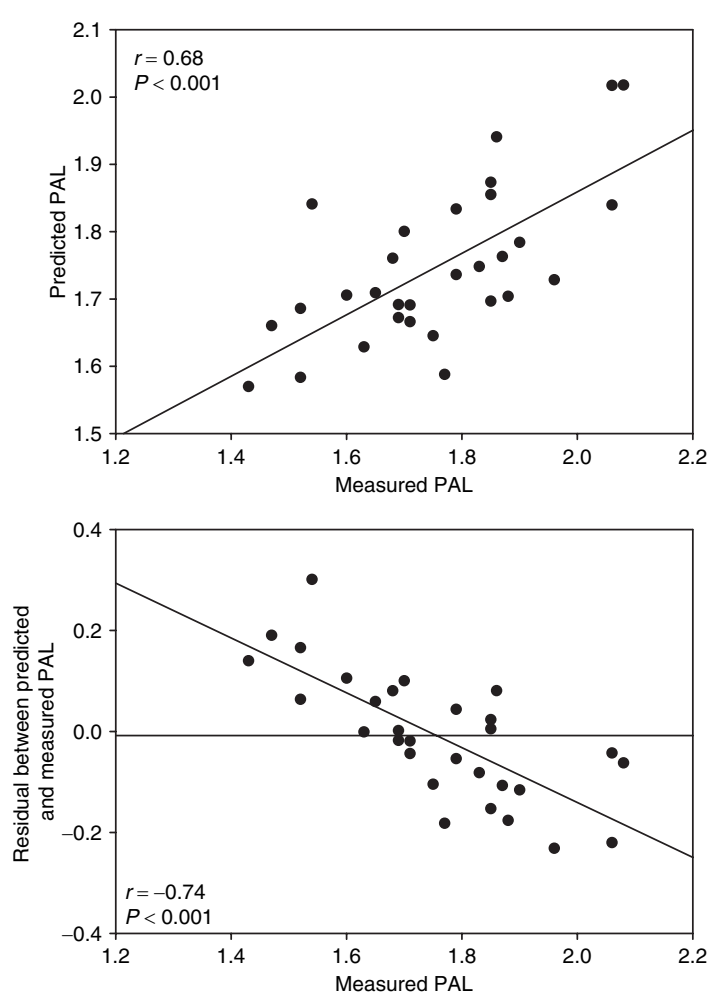

Figure 4 Regression and residual plots of the prediction models of physical activity level (PAL) based on accelerometer output.

measures of physical activity. The model developed to predict PAL showed an explained variation in PAL of $46 \%(r=0.68$, $P<0.001)$. The regression equation was given by:

$$
\mathrm{PAL}=1.354+256 \times 10^{-9} \times \mathrm{Cnts} / \mathrm{d} .
$$

The residuals of the model were negatively associated with the measured PAL $(r=-0.74, P<0.001)$ (Figure 4$)$. The SEE of the models was of 0.13 , representing $7.4 \%$ of the average PAL. The SEV was of 0.13 . The model developed to predict AEE $/ \mathrm{kg}$ explained $50 \%$ of the variation in $\mathrm{AEE} / \mathrm{kg}(r=0.71, P<0.001)$. The regression equation was given by:

$$
\mathrm{AEE} / \mathrm{kg}(\mathrm{MJ} / \mathrm{day} / \mathrm{kg})=0.0135+26 \times 10^{-9} \times \mathrm{Cnts} / \mathrm{d} .
$$

The residuals of this model were negatively associated with the measured AEE $/ \mathrm{kg}(r=-0.68, P<0.001)$. The SEE of the models was of $0.012 \mathrm{MJ} / \mathrm{day} / \mathrm{kg}$, representing $22 \%$ of the average $\mathrm{AEE} / \mathrm{kg}$. The SEV was of $0.013 \mathrm{MJ} / \mathrm{day} / \mathrm{kg}$.

\section{DISCUSSION}

The Tracmor $_{\mathrm{D}}$ is one of the smallest and light-weighted instruments developed to measure physical activity. The design of Tracmor $_{\mathrm{D}}$ was focused to reduce obtrusiveness and to diminish the interference of the measuring system with the individuals' activity behavior. Considering that important specifications of wearable activity monitors, such as the battery life and the onboard processing capacity, are often negatively affected by the miniaturization of the measuring system, we felt it important to test the ability of the Tracmor ${ }_{\mathrm{D}}$ accelerometer in predicting free-living energy expenditure.

The developed models used to predict TEE showed that Cnts/d measured with the Tracmor $_{D}$ significantly contributed to the explained variance in TEE. In particular, depending on which independent variables were considered to adjust for differences in body size, Cnts/d accounted for $17-23 \%$ of the explained variance in TEE of the models. Correspondingly, Cnts/d contributed for $16-23 \%$ to the explained variance in AEE. Furthermore, a direct linear association was observed between Cnts/d and PAL, and between Cnts/d and AEE/kg. Thus, in all developed models the contribution of the Tracmor ${ }_{D}$ was necessary to achieve a higher accuracy in predicting freeliving energy expenditure.

From the residual analysis of the prediction models a significant negative trend was observed. This means that applying the developed models to population of subjects with a very lowactivity level, and with low levels of TEE and AEE might produce an overestimation of PAL and energy expenditure. On the other hand, an underestimation of PAL and energy expenditure could be expected in subjects with a very high-activity level, and a correspondingly high TEE and AEE. However, the SEE of the TEE prediction models observed in the broad range of TEE of the present study was between 7 and $10 \%$. Furthermore, the study participants covered the range of normal daily life activity levels, as indicated by the measured PAL range which was from 1.43 to 2.08 , but they were not highly physically active. Considering that the obtained SEE in this wide range of PAL was $<7 \%$, we can assume that the observed trend in the residual would not limit the applicability of the PAL prediction model to a population of subjects within the range of normal activity levels. This trend in the residual could be due to an overestimation of the energy cost for sedentary activities that might characterize the behavior of subjects with a low PAL. Likewise, this trend could be caused by an underestimation of the energy cost for high-intensity activities, which might characterize the behavior of subjects with high PAL. However, specific studies are necessary to investigate the performance of the Tracmor in measuring energy expenditure for a number of activity types to understand which factors could be imputed of reducing the accuracy of the TEE, AEE, and PAL prediction models.

Only a small number of accelerometers have been validated in free-living conditions against the gold standard technique of DLW. Those that were validated, often show poor correlations with energy expenditure or the main contribution to the explained variation in TEE or AEE was determined by subjects' physical characteristics (10). These prediction performances are summarized in Table 4 . Indeed, one of the most popular and most frequently validated accelerometers, the Actigraph (latest size: $5.1 \times 4.1 \times 1.5 \mathrm{~cm}$; Actigraph, Fort Walton Beach, FL) released in several versions, showed poorer accuracy in predicting TEE, AEE, AEE $/ \mathrm{kg}$, and PAL as compared to the Tracmor $_{D}(10,23,26,27)$. Similarly, prediction models of PAL and AEE developed for the Tritrac-R3D (size: $12 \times 6.5 \times$ $2.2 \mathrm{~cm}$; Hemokinetics, Madison, WI) showed a lower accuracy as compared to the $\operatorname{Tracmor}_{\mathrm{D}}(10,28)$. Furthermore, a recent 
Table 4 Accuracy of different accelerometers in predicting TEE and AEE compared to doubly labeled water measures

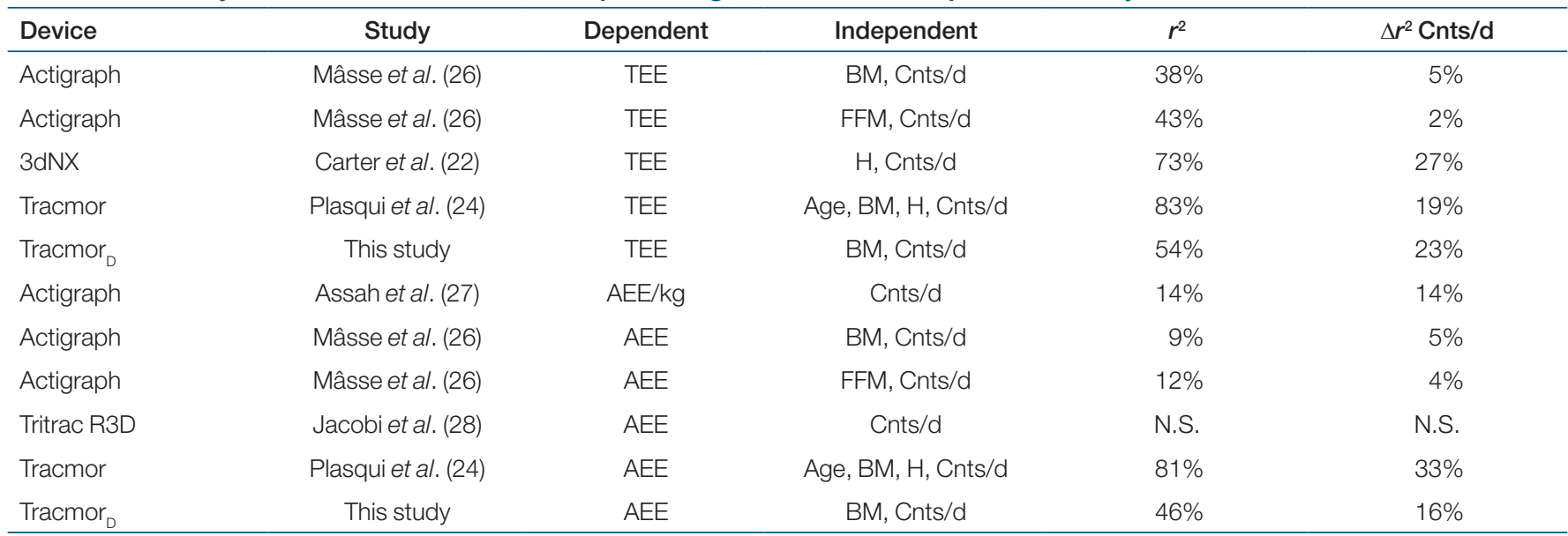

$\Delta r^{2} \mathrm{Cnts} / \mathrm{d}$, contribution to the correlation coefficient of the accelerometer output; AEE, activity energy expenditure; AEE/kg, AEE corrected by body mass; BM, body mass; Cnts/d, counts per day which represents the accelerometer output; dependent, predicted parameter; FFM, fat-free mass; $\mathrm{H}$, height; independent, parameters used in the prediction equation; N.S., not statistical significance; $r^{2}$, correlation coefficient of the model; TEE, total energy expenditure.

validation study investigated the ability of several prediction equations developed for the Actigraph and for the Tritrac-R3D to estimate TEE, as measured in free-living conditions using DLW (9). This study concluded that only 2 out of 14 prediction equations showed to produce a prediction of TEE which was nonsignificantly different from the measured TEE, but a large SEE was still observed (15-17\% of the mean TEE). However, it should be noted that these performances could have been worsened by the fact that an independent population was used for testing the accuracy of the prediction equations. Very few studies reported a higher accuracy in predicting free-living energy expenditure than the accuracy of the models obtained with the Tracmor ${ }_{D}$. Carter et al. (22) developed a model to predict TEE using as independent variables body height and activity counts as measured with the $3 \mathrm{dNX}$ accelerometer (size: $12.5 \times 5.8 \times 0.8 \mathrm{~cm}$; BioTel, Bristol, UK). However, the $3 \mathrm{dNX}$ accelerometer did not show any association with PAL neither with AEE/kg, which are parameters directly related to physical activity. Plasqui et al. (24) measured physical activity using an early version of the Tracmor accelerometer (size: $7.2 \times 2.6 \times 0.7 \mathrm{~cm}$; Philips Research Laboratories, Eindhoven, the Netherlands) and developed a prediction model of TEE using as independent variables SMR and activity counts. The explained variation of the model was $90 \%$. Using the Tracmor ${ }_{D}$, the model based on the same independent variables showed to explain the variation in TEE for $76 \%$. Furthermore, Plasqui et al. (24) developed a model to predict TEE using as independent variables age, body mass, height, and activity counts (Table 4). In our study, TEE was predicted by body mass and Cnts/d. This model explained $54 \%$ of the variation in TEE whereas Cnts/d accounted for $23 \%$ to the explained variation in TEE. Although comparing these prediction models is difficult because of the different independent variables included in the regression, it appeared that the ones developed in our study showed a lower explained variance in TEE. However, the contribution of Cnts/d to explain the variation in TEE was similar for the Tracmor and for the Tracmor ${ }_{D}$ (Table 4).
Improvements in the prediction accuracy of energy expenditure have been proposed by addressing specific issues related to accelerometer-based measurements of physical activity, such as the nonuniqueness in the relation between activity counts and the intraindividual variability in AEE $(14,29)$. For example, activities like running and cycling might generate a different amount of activity counts even at similar rates of AEE. For this reason, systems able to identify types of physical activity have been developed to correct for the dissimilar contribution to AEE of different activity types. The Intelligent Device for Energy Expenditure and Physical Activity (IDEEA) (MiniSun LLC, Fresno, CA) and the ActiReg (PreMed AS, Oslo, Norway) have been proposed to identify activity type and to determine AEE by considering which category of activity was performed by the subject $(15,30)$. These activity monitors are relatively complex systems: the IDEEA and the ActiReg are constituted by, respectively, 5 and 2 wire connected acceleration and tilt sensors, to be placed in different locations of the body. This highly reduces the wearability of the monitoring system and it might interfere with the individuals' activity behavior. However, recent advances in accelerometer-based activity monitoring allowed the development of methods to identify types of daily physical activity using a single triaxial accelerometer, such as sitting, standing, walking, running, and cycling (31), or to distinguish between locomotive and lifestyle activities (12). The IDEEA showed high accuracy for estimating TEE measured using indirect calorimetry during standardized activity trials or during stay in respiration chamber $(11,32)$. However, no data is available on the performance of this sophisticated activity monitor in free-living conditions. Conversely, the ActiReg has been validated against DLW, but the accuracy of the model in predicting TEE $\left(r^{2}=64 \%\right)$ was comparable to that observed in studies in which much simpler activity monitors were used to monitor physical activity (15).

A limitation of this study could be identified in the fact that the most accurate TEE prediction model was based on the measured SMR to account for differences in body size. Considering that measuring SMR requires a consistent effort, 
this accurate prediction model might have limited applicability in large-population studies. It should also be noted that even if the contribution of the Tracmor and of the Tracmor ${ }_{D}$ in predicting energy expenditure was similar, the Tracmor $_{D}$ might operate differently than the Tracmor, for instance by adopting energy-saving strategies for recording the acceleration signal. Furthermore, the two instruments are equipped with a different acceleration sensor. Indeed, as the Tracmor is based on the piezo-electric technology, the Tracmor $_{D}$ is equipped with a piezo-capacitive accelerometer, which allows the detection of both dynamic and static accelerations.

In conclusion, this study showed that the $\operatorname{Tracmor}_{\mathrm{D}}$ is a highly accurate instrument for predicting free-living energy expenditure. TEE could be explained for $76 \%$ when SMR and $\mathrm{Cnts} / \mathrm{d}$ were included in the prediction model. Although the Tracmor $_{D}$ is smaller, lighter, and less energy consuming than several other activity monitors, the measured Cnts/d significantly contributed to the explained variance in TEE, which was similar to that reported for the Tracmor accelerometer. This means that the improvement in miniaturization did not harm the ability of the Tracmor ${ }_{D}$ in collecting information of the body acceleration to correctly quantify physical activity for determining TEE, AEE, and PAL.

\section{ACKNOWLEDGMENTS}

The study was performed in collaboration with Philips Research Laboratories (Eindhoven, the Netherlands) and we thank Philips Research Laboratories for their financial support and Philips New Wellness Solutions (Lifestyle Incubator, the Netherlands) for providing the Tracmor $_{D}$ instruments.

\section{DISCLOSURE}

This study was funded by Philips Research Laboratories.

(c) 2010 The Obesity Society

\section{REFERENCES}

1. WHO. Obesity: preventing and managing the global epidemic. World Health Organ Tech Rep Ser 2000;894:1-253.

2. Must A, Spadano J, Coakley EH et al. The disease burden associated with overweight and obesity. JAMA 1999;282:1523-1529.

3. Joosen AM, Gielen M, Vlietinck R, Westerterp KR. Genetic analysis of physical activity in twins. Am J Clin Nutr 2005;82:1253-1259.

4. Hill JO, Wyatt HR, Reed GW, Peters JC. Obesity and the environment: where do we go from here? Science 2003;299:853-855.

5. Haskell WL, Lee IM, Pate RR et al. Physical activity and public health: updated recommendation for adults from the American College of Sports Medicine and the American Heart Association. Med Sci Sports Exerc 2007;39:1423-1434.

6. Johansson G, Westerterp KR. Assessment of the physical activity level with two questions: validation with doubly labeled water. Int J Obes (Lond) 2008;32:1031-1033.

7. Brage S, Brage N, Ekelund $U$ et al. Effect of combined movement and heart rate monitor placement on physical activity estimates during treadmill locomotion and free-living. Eur J Appl Physiol 2006;96:517-524.

8. Chen KY, Bassett DR Jr. The technology of accelerometry-based activity monitors: current and future. Med Sci Sports Exerc 2005;37:S490-S500.

9. Leenders NY, Sherman WM, Nagaraja HN, Kien CL. Evaluation of methods to assess physical activity in free-living conditions. Med Sci Sports Exerc 2001;33:1233-1240.
10. Plasqui G, Westerterp KR. Physical activity assessment with accelerometers: an evaluation against doubly labeled water. Obesity (Silver Spring) 2007;15:2371-2379.

11. Rothney MP, Neumann M, Béziat A, Chen KY. An artificial neural network model of energy expenditure using nonintegrated acceleration signals. J Appl Physiol 2007;103:1419-1427.

12. Crouter SE, Clowers KG, Bassett DR Jr. A novel method for using accelerometer data to predict energy expenditure. J Appl Physiol 2006;100:1324-1331.

13. Chen $\mathrm{KY}$, Sun $\mathrm{M}$. Improving energy expenditure estimation by using a triaxial accelerometer. J Appl Physiol 1997;83:2112-2122.

14. Bonomi AG, Plasqui G, Goris AH, Westerterp KR. Improving assessment of daily energy expenditure by identifying types of physical activity with a single accelerometer. J Appl Physiol 2009;107:655-661.

15. Hustvedt BE, Christophersen A, Johnsen LR et al. Description and validation of the ActiReg: a novel instrument to measure physical activity and energy expenditure. Br J Nutr 2004;92:1001-1008.

16. Zakeri I, Adolph AL, Puyau MR, Vohra FA, Butte NF. Application of cross-sectional time series modeling for the prediction of energy expenditure from heart rate and accelerometry. J Appl Physiol 2008;104:1665-1673.

17. Westerterp KR, Wouters L, van Marken Lichtenbelt WD. The Maastricht protocol for the measurement of body composition and energy expenditure with labeled water. Obes Res 1995;3(Suppl 1):49-57.

18. Siri WE. Body composition from fluid spaces and density: analysis of methods. 1961. Nutrition 1993;9:480-91; discussion 480, 492.

19. Schoffelen PF, Westerterp KR, Saris WH, Ten Hoor F. A dual-respiration chamber system with automated calibration. J Appl Physiol 1997;83: 2064-2072.

20. WEIR JB. New methods for calculating metabolic rate with special reference to protein metabolism. J Physiol (Lond) 1949;109:1-9.

21. Westerterp KR. Diet induced thermogenesis. Nutr Metab (Lond) 2004;1:5.

22. Carter J, Wilkinson D, Blacker S et al. An investigation of a novel threedimensional activity monitor to predict free-living energy expenditure. J Sports Sci 2008;26:553-561.

23. Ekelund U, Yngve A, Brage S, Westerterp K, Sjöström M. Body movement and physical activity energy expenditure in children and adolescents: how to adjust for differences in body size and age. Am J Clin Nutr 2004;79: 851-856.

24. Plasqui G, Joosen AM, Kester AD, Goris AH, Westerterp KR. Measuring free-living energy expenditure and physical activity with triaxial accelerometry. Obes Res 2005;13:1363-1369.

25. Goris AH, Holmes R. The effect of a Lifestyle Activity intervention program on improving physical activity behavior of employees. In: PERSUASIVE 2008. Springer Berlin: Oulu, Finland, 2008, June 04-06.

26. Mâsse LC, Fulton JE, Watson KL et al. Influence of body composition on physical activity validation studies using doubly labeled water. J Appl Physiol 2004:96:1357-1364.

27. Assah FK, Ekelund U, Brage $S$ et al. Predicting physical activity energy expenditure using accelerometry in adults from sub-Sahara Africa. Obesity (Silver Spring) 2009;17:1588-1595.

28. Jacobi D, Perrin AE, Grosman N et al. Physical activity-related energy expenditure with the RT3 and TriTrac accelerometers in overweight adults. Obesity (Silver Spring) 2007;15:950-956.

29. van Hees VT, van Lummel RC, Westerterp KR. Estimating activityrelated energy expenditure under sedentary conditions using a tri-axial seismic accelerometer. Obesity (Silver Spring) 2009;17: 1287-1292.

30. Zhang K, Werner P, Sun M, Pi-Sunyer FX, Boozer CN. Measurement of human daily physical activity. Obes Res 2003;11:33-40.

31. Bonomi AG, Goris AH, Yin B, Westerterp KR. Detection of type, duration, and intensity of physical activity using an accelerometer. Med Sci Sports Exerc 2009;41:1770-1777.

32. Zhang K, Pi-Sunyer FX, Boozer CN. Improving energy expenditure estimation for physical activity. Med Sci Sports Exerc 2004;36: 883-889. 